OPEN ACCESS

Edited by:

Alla Lapidus,

Saint Petersburg State University,

Russia

Reviewed by:

Jens Andre Hammerl,

Bundesinstitut für Risikobewertung,

Germany

Peter S. Evans,

Food Safety and Inspection Service

(USDA), United States

Lucas Harrison,

United States Food and Drug

Administration, United States

*Correspondence:

Maria Hoffmann

maria.hoffmann@fda.hhs.gov

Specialty section:

This article was submitted to Evolutionary and Genomic Microbiology,

a section of the journal

Frontiers in Microbiology

Received: 14 October 2020 Accepted: 13 January 2021

Published: 11 February 2021

Citation:

Yao K, González-Escalona N and Hoffmann M (2021) Multiple Displacement Amplification as a Solution for Low Copy Number Plasmid Sequencing.

Front. Microbiol. 12:617487. doi: 10.3389/fmicb.2021.617487

\section{Multiple Displacement Amplification as a Solution for Low Copy Number Plasmid Sequencing}

\author{
Kuan Yao, Narjol González-Escalona and Maria Hoffmann*
}

Division of Microbiology, Center for Food Safety and Applied Nutrition, Food and Drug Administration, College Park, MD, United States

Plasmids play a major role in bacterial adaptation to environmental stress and often contribute to antibiotic resistance and disease virulence. Although the complete sequence of each plasmid is essential for studying plasmid biology, most antibiotic resistance and virulence plasmids in Salmonella are present only in a low copy number, making extraction and sequencing difficult. Long read sequencing technologies require higher concentrations of DNA to provide optimal results. To resolve this problem, we assessed the sufficiency of multiple displacement amplification (MDA) for replicating Salmonella plasmid DNA to a satisfactory concentration for accurate sequencing and multiplexing. Nine Salmonella enterica isolates, representing nine different serovars carrying plasmids for which sequence data are already available at $\mathrm{NCBI}$, were cultured and their plasmids isolated using an alkaline lysis extraction protocol. We then used the Phi29 polymerase to perform MDA, thereby obtaining enough plasmid DNA for long read sequencing. These amplified plasmids were multiplexed and sequenced on one single molecule, real-time (SMRT) cell with the Pacific Biosciences (Pacbio) Sequel sequencer. We were able to close all Salmonella plasmids (sizes ranged from 38 to $166 \mathrm{~Kb}$ ) with sequencing coverage from 24 to 2,582X. This protocol, consisting of plasmid isolation, MDA, and multiplex sequencing, is an effective and fast method for closing high-molecular weight and low-copy-number plasmids. This high throughput protocol reduces the time and cost of plasmid closure.

Keywords: Salmonella, plasmid, sequencing, PacBio, multiplexing

\section{INTRODUCTION}

Salmonella enterica is a Gram-negative foodborne pathogen associated with 1.2 million foodborne illnesses, 23,000 hospitalizations, and 450 deaths in the United States every year (Scallan et al., 2011). Due to selective pressures, prevalence of Salmonella isolates resistant to fluoroquinolones and third generation cephalosporin has been increasing in both clinical and agriculture settings (Karp et al., 2017). The National Antimicrobial Resistance Monitoring System (NARMS) has observed multidrug resistance Salmonella serotype doubling between the years 2011 and 2015 (CDC, 2018). The emergence of new isolates that are resistant to antibiotics, antimicrobials, sanitizers, and heavy metals complicates surveillance and prevention efforts worldwide. These forms of resistance also reduce the available methods for treating patients who contract resistant forms of salmonellosis. 
Resistance can disseminate across serovars through mobile elements such as plasmids. Bacterial plasmids can carry genes conveying resistance to antibiotics, sanitizers, and/or heavy metals, as well as elements that enable toxin production, such as ehxA (Lindsey et al., 2009; Lorenz et al., 2016). In 2009, NARMS surveillance detected Salmonella Heidelberg isolates displaying extended-spectrum cephalosporin (ESC) resistance; these were found to be carrying plasmids encoding bla(CMY) beta-lactamase (Folster et al., 2012). More recent research has identified multidrug resistant Salmonella Infantis harboring $m c r-1$ and $b l a_{\mathrm{CTZ}-\mathrm{M}-1}$ genes in their plasmids (Carfora et al., 2018). The mobility of plasmids allows isolates to easily acquire new genes through conjugation, transposition, and site-specific recombination; once a plasmid is acquired, selection pressures may establish these traits throughout the population (Bennett, 2009; Liao et al., 2019).

Understanding the evolution of antimicrobial resistant (AMR) pathogens and the spread of mobile genetic elements which convey resistance, requires studying fully-closed sequences of plasmids. However, although whole genome sequencing (WGS) has become a reliable and comprehensive method for tracing the evolution of AMR pathogens (Hoffmann et al., 2014; Allard et al., 2016), plasmids have not received the same level of attention as chromosomal DNA. One of the obstacles to effectively obtain complete closed plasmid sequences is the usual multiple repetitive regions found on plasmids (e.g., insertion sequences) which make assembly with short reads difficult. Another obstacle is having enough initial DNA material to perform accurate sequencing using long read sequencing platforms, as larger plasmids typically exist in low copy numbers inside the cell.

One method for acquiring high molecular weight, low copy number plasmid DNA for sequencing on the Pacific Biosciences (Pacbio) sequencing platform was published by Hoffmann et al. (2017). Those authors obtained sufficient quantity of Salmonella plasmids using a modified plasmid mini preparation and then transformed the isolated plasmids by electroporation into Escherichia coli $\mathrm{DH} 10 \mathrm{Br}$ in order to increase plasmid copy number. Then, the Qiagen Large-Construct kit ${ }^{\mathrm{TM}}$ (Qiagen, Gaithersburg, MD) was used to extract highly concentrated plasmid DNA suitable for sequencing with the Pacbio RSII (Pacific Biosciences, Menlo Park, CA, United States). This process allowed to completely close high molecular weight, low-copy number plasmids for Salmonella. However, any method that requires several steps of isolation and transformation into E. coli, consumes both laboratory resources and time, therefore a simpler, more efficient, and cost-effective method for closing high molecular weight, low copy number plasmids will be desirable.

In this report, we explore a different method for increasing the amount of plasmid DNA available for sequencing. We tested a method known as multiple displacement amplification (MDA; Cheung and Nelson, 1996), to replicate high molecular weight, low copy number plasmid DNA. Results obtained using MDA are very uniform across the target and have far less amplification bias compared to the traditional PCR-based whole genome amplification (WGA) technologies (Dean et al., 2002;
Spits et al., 2006; Bleier et al., 2008). In contrast to PCR-based WGA technologies, Phi29 polymerase has $3^{\prime} \rightarrow 5^{\prime}$ exonuclease proofreading activity and maintains a much higher fidelity compared to Taq DNA polymerase during replication (Spits et al., 2006).

The process of MDA is a natural occurring method of replicating genetic material, used by some Gram-negative bacteria and some species of Archaea (del Solar et al., 1998). This simple mechanism is an exponential process, making it a very useful technique for amplifying circular DNA in the laboratory (Dean et al., 2002). Here, we show how switching from more labor-intensive methods of isolating and amplifying plasmid DNA to the extremely efficient MDA process will allow researchers to quickly acquire high molecular weight, low copy number plasmid DNA from foodborne pathogens in sufficient amounts, and of appropriate quality, for third generation sequencing techniques. By selecting bacteria whose plasmids have previously been sequenced, we will be able to determine the fidelity of amplification using MDA.

\section{MATERIALS AND METHODS}

\section{Bacterial Isolates}

We selected nine $S$. enterica isolates representing nine different serovars. All isolates had been previously sequenced using Illumina short read and Pacific Bioscience long read sequence technology, analyzed, and their data deposited in the NCBI GenBank database. All isolates carried either one or two high molecular weight, low copy number plasmids, which ranged in size from 34,175 to $166,496 \mathrm{bp}$. The complete list of serovars and their metadata including accession numbers are listed in Table 1, and the complete reference sequences can be obtained from NCBI GenBank under https://www.ncbi.nlm.nih.gov/ nucleotide/.

\section{Plasmid DNA Extraction}

The workflow, beginning from bacterial culture and ending with producing concentrated, high molecular weight plasmid DNA, is summarized in Figure 1. The nine isolates were cultured separately in Luria Bertani broth (LB; Becton Dickinson, Franklin Lakes, NJ) overnight at $37^{\circ} \mathrm{C}$, and plasmid DNA was extracted using an alkaline lysis extraction protocol previously reported by Hoffmann et al. (2017). Specifically, bacterial cells from overnight cultures were harvested by centrifugation at $6,000 \mathrm{~g}$ for $10 \mathrm{~min}$ at $4^{\circ} \mathrm{C}$. The pellet for each isolate was resuspended in $350 \mu$ of resuspension buffer containing: 1X Phosphate-buffered saline (ThermoFisher, Waltham, MA, United States), $40 \mathrm{mM}$ Tris at $\mathrm{pH} 8$ (ThermoFisher, Waltham, MA, United States), $1 \mathrm{mM}$ EDTA (ThermoFisher, Waltham, MA, United States), and $200 \mu \mathrm{g}$ of RNase A (Qiagen, Hilden, Germany). Three hundred and fifty microliter of lysis buffer containing $0.05 \mathrm{~N} \mathrm{NaOH}$ (ThermoFisher, Waltham, MA, United States) and 0.25\% SDS (ThermoFisher, Waltham, MA, United States) was added to the bacterial mixture and mixed by gentle inversion. After 2 -min incubation of the lysis buffer at $25^{\circ} \mathrm{C}, 500 \mu \mathrm{l}$ of chilled 


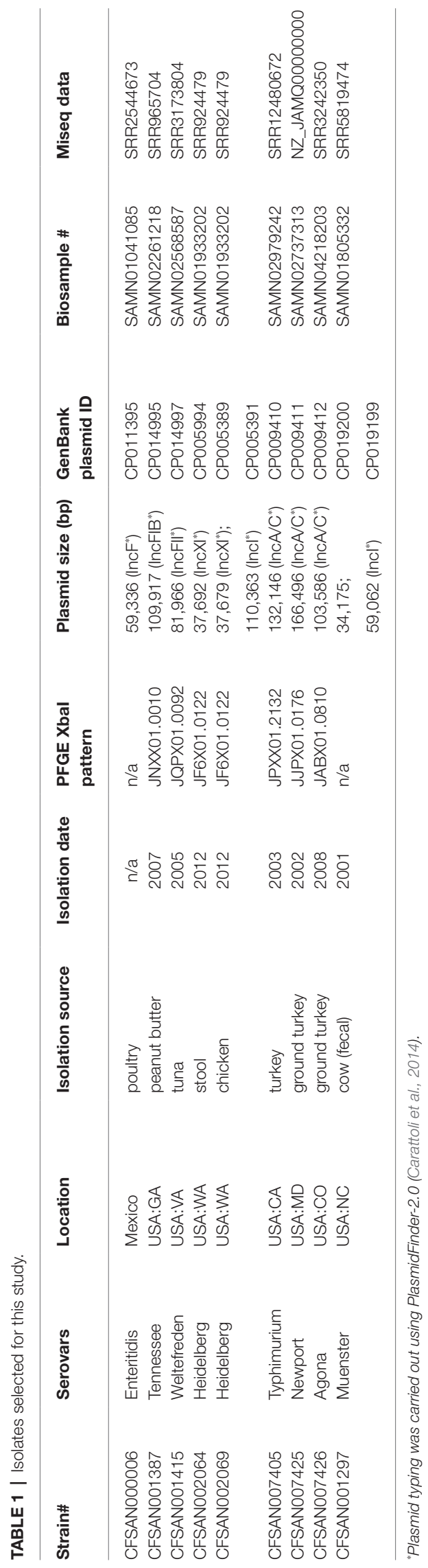

1.32 M potassium acetate buffer (ThermoFisher, Waltham, MA, United States) was mixed into the solution and incubated for an additional $5 \mathrm{~min}$ to neutralize the reaction. The supernatant was extracted after centrifugation at $20,800 \mathrm{~g}$ for $10 \mathrm{~min}$ at $4^{\circ} \mathrm{C}$. An equal amount of phenol:chloroform:isoamyl alcohol solution (25:24:1; ThermoFisher, Waltham, MA, United States) was mixed with the supernatant until the solution had a homogenous milky appearance. This solution was centrifuged at $20,800 \mathrm{~g}$ for $8 \mathrm{~min}$ at $4^{\circ} \mathrm{C}$. The clear top aqueous layer was taken and centrifuged at 20,800 $g$ for $10 \mathrm{~min}$ at $4^{\circ} \mathrm{C}$.

The supernatant containing plasmid DNA was transferred into a clean $1.5 \mathrm{ml}$ tube. That DNA was precipitated by adding 0.1 volume of $3 \mathrm{M}$ sodium acetate (ThermoFisher, Waltham, MA, United States) and $500 \mu \mathrm{l}$ of $100 \%$ isopropanol (SigmaAldrich, St. Louis, MO, United States). The solution was mixed thoroughly by inversion and centrifuged at 20,800 $\mathrm{g}$ for $10 \mathrm{~min}$ at $4^{\circ} \mathrm{C}$. The supernatant was discarded and the plasmid DNA pellet was washed twice with $1 \mathrm{ml}$ of $70 \%$ ethanol (SigmaAldrich, St. Louis, MO, United States) and centrifuged at $20,800 \mathrm{~g}$ for $5 \mathrm{~min}$. After air drying the plasmid pellet for $5 \mathrm{~min}$, it was re-suspended in $12 \mu \mathrm{l}$ ultra-pure DNase/RNase free distilled water (Thermo Fisher Scientific, MA, United States). Concentrations of plasmid DNA were measured using Qubit 3.0 fluorometer (Thermo Fisher Scientific, MA, United States) following the manufacturer's protocol.

\section{Chromosomal DNA Digestion}

Chromosomal DNA was removed from the isolated plasmids using Plasmid-Safe ATP-Dependent DNase (Epicenter, Madison, WI, United States) following the manufacturer's preparation recommendations. First, excess enzymes were removed from the plasmid DNA solution through ethanol precipitation. About $0.5 \mu \mathrm{l}$ of $3 \mathrm{M}$ sodium acetate (ThermoFisher, Waltham, MA, United States) and $140 \mu \mathrm{l}$ of cold $100 \%$ ethanol (Sigma-Aldrich, St. Louis, MO, United States) were added to the DNase treated plasmid DNA. The sample was then incubated at $-20^{\circ} \mathrm{C}$ for $60 \mathrm{~min}$ and centrifuged at $20,800 \mathrm{~g}$ for $20 \mathrm{~min}$ at $4^{\circ} \mathrm{C}$. After the supernatant was discarded, the pellet was washed twice with $1 \mathrm{ml}$ of $70 \%$ ethanol and the plasmid DNA was re-dissolved in $20 \mu \mathrm{l}$ ultra-pure DNase/RNase free distilled water (ThermoFisher, Waltham, MA, United States).

\section{Multiple Deplacement Amplification and DNA Size Selection}

Once the plasmids had been extracted, we used the REPLI-g Mini kit (Qiagen, Hilden, Germany) to perform MDA (three replicates) for each set of plasmid DNA obtained.

We assessed the quality and the size of the plasmid DNA using the Fragment Analyzer DNF-464 High Sensitivity Large Fragment $50 \mathrm{~Kb}$ analysis kit (Advanced Analytical Technologies, Inc., Ankeny, IA, United States). When the results of the Fragment Analyzer showed that an isolate carries both high molecular weight, low copy number plasmids along with low molecular weight, high copy number plasmids, size selection was performed. We used the BluePippin system (Sage Science, Inc., Beverly, 


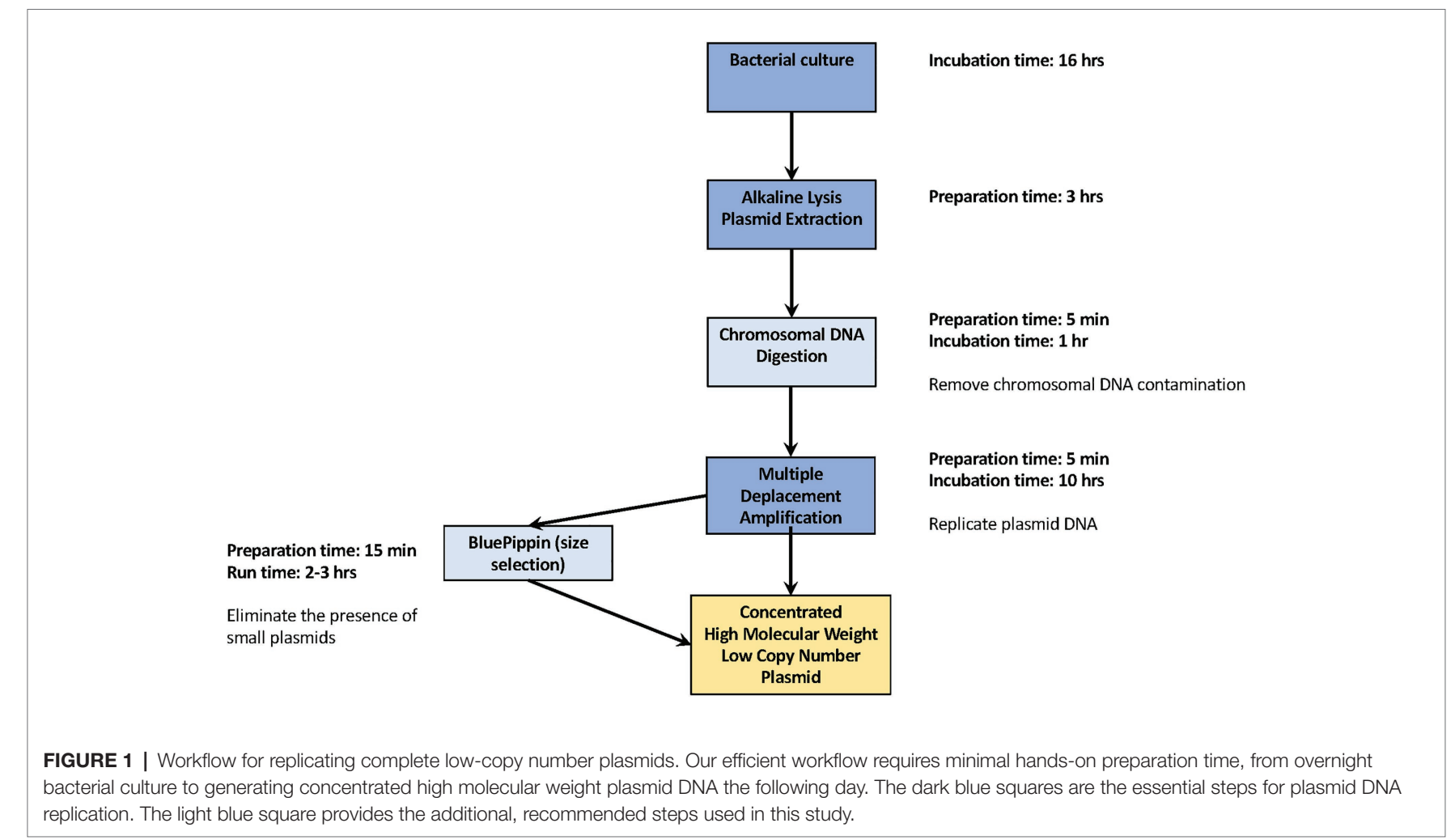

MA, United States) to select for fragment sizes between 6 and $50 \mathrm{~kb}$ on a Free Marker S1 High-pass 6-10 Kb vs. three Agarose Gel Cassette (Sage Science, Inc., Beverly, MA, United States). All MDA products (size selected and non-size selected) were purified using Agencourt AMPure XP beads (Beckman Coulter), according to the manufacturer's clean-up protocol. The concentrations of the purified MDA products were measured using Qubit 3.0 fluorometer (Thermo Fisher Scientific, MA, United States) following the manufacturer's protocol.

\section{DNA Library Construction and Multiplex Sequencing}

Amplified and purified plasmid DNA from nine isolates were used to construct multiplexed microbial SMRTbell libraries using the SMRTbell Template Prep Kit 1.0 (PacBio, Menlo Park, CA) according to the manufacturer's protocol. Each isolate was ligated with a unique barcode using the SMRTbell Barcoded Adapter Complete Prep Kit-96 (PacBio, Menlo Park, CA). The barcoded adapters chosen for the study (listed in Table 2) were recommended by Pacific Biosciences for microbial multiplexing. These multiplexed SMRTbell libraries were then sequenced on a PacBio Sequel sequencer (PacBio, Menlo Park, CA) using PacBio Sequel V2.0 chemistry on one Sequel single molecule, real-time (SMRT) cell $1 \mathrm{M}$ v2 (PacBio, Menlo Park, CA), with a 600-min collection time.

\section{Sequence Analysis}

Raw sequencing data were demultipexed by running the Demultiplex Barcodes application with symmetric mode in
SMRTLink v.5.1.0. (PacBio, Menlo Park, CA). The adapter sequences (Table 2) were trimmed and filtered out during the demultiplexing process. De novo assembly for each isolate was done using the PacBio hierarchical genome assembly process HGAP4.0 (Chin et al., 2013). We adjusted the target genome size in the assembly filter for each corresponding plasmid size while the other assembly parameters were set to Default. To check for genome closure, the overlapping regions were accessed using Gepard (Krumsiek et al., 2007). The plasmid genomes were aligned against their reference genomes obtained from GenBank. MAUVE aligner version 20150226 using progressive algorithm with default settings (Darling et al., 2004) was used to align each replicate against one other to assess the presence of variance.

\section{RESULTS AND DISCUSSION}

We developed a protocol for replicating low-copy number plasmid DNA in sufficient concentrations and quality to successfully close plasmid genomes. We then evaluated that protocol using three experimental replicates of nine Salmonella isolates to identify potential amplification biases that could occur during the MDA process. Our basic method of replicating low copy-number plasmid DNA requires as little as $3 \mathrm{~h}$ of hands-on time, providing only the essential steps for plasmid extraction and MDA (marked in dark blue in Figure 1) are performed. However, if you wish to acquire higher quality products for low copy number plasmids along with low molecular weight, our recommended additional steps, chromosomal DNA 
TABLE 2 | Barcoded adapter names and sequences.

\begin{tabular}{|c|c|c|c|}
\hline Isolate serovars & CFSAN\# & Barcoded adapter name & Barcoded adapter Sequence \\
\hline Enteritidis & CFSAN000006 & BC1002 & CTCACAGTCTGTGTGT \\
\hline Tennessee & CFSAN001387 & BC1016 & ATACTATCTCTCTATG \\
\hline Weltefreden & CFSAN001415 & BC1032 & CACTATCTCTAGTCTC \\
\hline Heidelberg & CFSAN002064 & BC1048 & GAGTGTGAGTGCACAC \\
\hline Heidelberg & CFSAN002069 & BC1100 & ACTACTGAGACATAGA \\
\hline Typhimurium & CFSAN007405 & BC1101 & TATATCGCGTCGCTAT \\
\hline Newport & CFSAN007425 & BC1055 & GATGAGATCTCGTGTG \\
\hline Agona & CFSAN007426 & BC1063 & AGTGTGTCATGCGTGT \\
\hline Muenster & CFSAN001297 & BC1118 & AGTATCATGTGTATCT \\
\hline
\end{tabular}

digestion and DNA size selection, requires only 20 more minuites hands-on time (marked in light blue in Figure 1). The higher the quality of the plasmid DNA, the more suitable it is for sequencing.

The size selection step is crucial for isolates that carry a larger plasmid along with a smaller plasmid, as size selection eliminates plasmids below $6 \mathrm{~kb}$ from the DNA to be amplified. Smaller plasmids are usually present in high copy number, therefore their DNA quantity will spike up during replication. That will cause problems during sequencing since most DNA will be amplified of the small plasmid DNA and only a small portion of the larger plasmid DNA of interest and therefore, the sequence data of the smaller plasmid will have a much higher coverage (data not shown). We assessed the quantity of plasmid DNA present after chromosomal DNA digestion treatment, and after performing the MDA (Figure 2). The average total plasmid DNA after chromosomal DNA digestion was $0.713 \mu \mathrm{g}$ (range 0.3-1.7 $\mu \mathrm{g}$ ), which includes also the amount of the smaller plasmid DNA (below $6 \mathrm{~kb}$ ). After MDA, we found the total amount of purified plasmid DNA increased in average up to $14.5 \mu \mathrm{g}$ (range 6-42 $\mu \mathrm{g}$ ) which is an average increase of 20 -fold and clearly shows that the new designed protocol is very effective. Hoffmann et al. (2017) obtained $\sim 20 \mu \mathrm{g}$ of high quality Salmonella plasmid DNA using their modified plasmid isolation protocol including transformation with E. coli $\mathrm{DH} 10 \mathrm{Br}$. The authors mentioned that at that time a minimum of $5 \mu \mathrm{g}$ DNA was required for sequencing on the Pacbio RS II. Therefore, the goal of using MDA is to produce at least $5 \mu \mathrm{g}$ DNA, which is successful as shown in Figure 2.

Having established that our method increased the quantity of plasmid DNA, we also assessed the quality of the DNA after amplification. Each amplification product was run on the Fragment Analyzer. The majority of plasmid DNA fragments obtained after MDA were above $30 \mathrm{~kb}$, with average fragment sizes ranging from 35 to $45 \mathrm{~kb}$. When the results of the Fragment Analyzer showed that an isolate carries both high molecular weight, low copy number plasmids along with low molecular weight, high copy number plasmids, it is important to perform size selection. The size selection is necessary in order to eliminate the low molecular weight, high copy number plasmids since they interfere with the results to achieve a high concentration for the high molecular weight, low copy number plasmids during MDA (experimental data not shown).
In order to assess whether any mutations or chimeras caused by the Phi29 DNA polymerase had been introduced during the MDA process, we performed three replicates of the MDA for all nine plasmid extraction products. Subsequently for each MDR product, a SMRTbell library was contructed and sequenced on the Pacbio Sequel sequencer. Although the total sequencing output, including all unbarcoded reads for each replicate, was 4.03, 2.39, and $1.69 \mathrm{~Gb}$, after demultiplexing and sequencing read filtering, the actual barcoded sample output decreased to $1.2 \mathrm{~Gb}$ and 717 and $608 \mathrm{Mb}$. Using the PacBio hierarchical genome assembly process HGAP4.0, we performed de novo assemblies on 11 plasmid genomes from nine isolates for all three replicates (total $=33$ assemblies). The assembled plasmid sizes and the corresponding coverage for each experimental replicate are displayed in Table 3. Our study included two isolates, S. Heidelberg CFSAN002069 and S. Muenster, that both carried two plasmids. For $S$. Muenster, two plasmid sizes were fairly close -34 and $59 \mathrm{~kb}$; whereas $S$. Heidelberg CFSAN002069 carried one plasmid of $38 \mathrm{~kb}$ and another one of $110 \mathrm{~kb}$. We obtained almost 2-fold coverage difference between the plasmids from S. Muenster (1,003 and 1779X), but found a 10 -fold coverage difference between the $S$. Heidelberg plasmids (2,220 and 268X). When plasmids were close in size, there were less preferential amplification within the sample. Three (pCFSAN007405, pCFSAN007425, and pCFSAN007426) of the 11 plasmids from our study were previously included in the study from Hoffmann et al. (2017). At that time, the authors obtained for CFSAN007426 very similar sequence coverage and for the other two plasmids two to three times higher sequence coverage. However, at that time, the multiplexing protocol was not used instead each plasmid was sequenced seperately. In general, we generated very high coverage for each plasmid, therefore, it is possible to multiplex at least 10 times more plasmid DNA, and the cost for plasmid sequencing is reduced by multiplexing.

Two features of the sequencing performance may be worth elaborating: low sequencing output and the large variability in sequencing coverage. At the time (early 2018) when these experiments were performed, PacBio only offered Sequel V2.0 chemistry and Sequel SMRT cell $1 \mathrm{M}$ v2. The microbial multiplexing barcode adaptor kit had not yet been released. Therefore, multiplexing protocol for greater than $10 \mathrm{~kb}$ libraries had not yet been fully optimized. Given these conditions, our actual sequencing output was less than optimal (4 Gb at best). After read filtering for barcode adapters, most of 


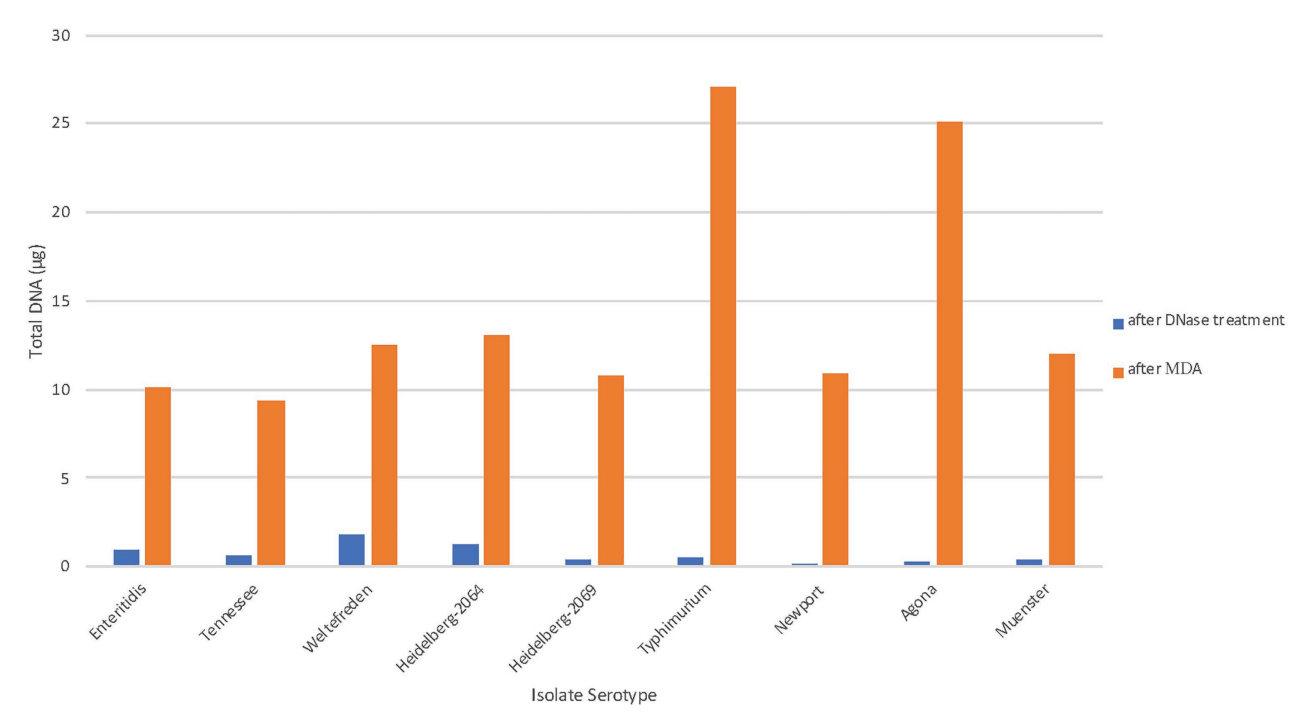

FIGURE 2 | Total plasmid DNA $(\mu \mathrm{g})$ after MDA. The total amounts of plasmid DNA in $\mu \mathrm{g}$ were measured at two different stages of the workflow. The blue bars represent the purified plasmid DNA after chromosomal digestion; the orange bar represent the total amplified DNA after MDA.

TABLE 3 | Plasmid size and sequence coverage for each replicate.

\begin{tabular}{|c|c|c|c|c|c|c|}
\hline \multirow[b]{2}{*}{ Isolate serovars } & \multicolumn{2}{|c|}{ Replicate 1} & \multicolumn{2}{|c|}{ Replicate 2} & \multicolumn{2}{|c|}{ Replicate 3} \\
\hline & Plasmid size (bp) & Coverage & Plasmid size (bp) & Coverage & Plasmid size (bp) & Coverage \\
\hline Enteritidis & 59,372 & $1,177 X$ & 59,372 & $1,235 x$ & 59,372 & $499 x$ \\
\hline Tennessee & 109,917 & $1,349 x$ & 109,917 & $461 X$ & 109,917 & $458 X$ \\
\hline Weltefreden & 81,966 & $2011 X$ & 81,966 & $1,028 x$ & 81,967 & $553 X$ \\
\hline Heidelberg CFSAN002064 & 37,692 & $66 X$ & 37,692 & $24 X$ & 37,692 & $25 x$ \\
\hline Heidelberg & 110,363 & $268 X$ & 110,363 & $289 x$ & 110,364 & $145 X$ \\
\hline Typhimurium & 132,147 & $190 \mathrm{X}$ & 132,149 & $223 x$ & 132,149 & $120 \mathrm{X}$ \\
\hline Newport & 166,506 & $417 X$ & 166,504 & $334 X$ & 166,505 & $323 x$ \\
\hline Agona & 103,587 & $2,582 x$ & 103,588 & $787 X$ & 103,587 & $973 x$ \\
\hline \multirow[t]{2}{*}{ Muenster } & 59,069 & $1779 x$ & 59,069 & $921 x$ & 59,069 & $661 x$ \\
\hline & 34,175 & $1,003 x$ & 34,175 & $726 x$ & 34,175 & $939 x$ \\
\hline
\end{tabular}

the unbarcoded reads and low quality reads were excluded. However, this left us with significantly less $(\sim 70 \%)$ data for de novo assembly. In the case of $S$. Heidelberg CFSAN002064, the sequencing coverage was decreased by (a) low total sequencing output, and (b) read filtering. Since then, PacBio had made significant upgrade in their chemistry and barcoded adaptor kit. New Sequel V3.0 chemistry can provide much higher sequencing output $(\sim 8 \mathrm{~Gb})$ and better barcode recognition (Pacific-Bioscience, 2019).

After analyzing the sequence data, the results were compared with the published sequences for each plasmid that had previously been archived in the NCBI GenBank. In contrast to PCR-based WGA technologies, Phi29 polymerase has $3^{\prime} \rightarrow 5^{\prime}$ exonuclease proofreading activity and maintains up to 1,000-fold higher fidelity compared to Taq DNA polymerase during replication. In order to detect sequencing single nucleotide polymorphisms (SNPs) or variants, we aligned the assembled genomes against each other using
Mauve (Table 4). Out of the 33 assembled plasmids, 24 did not have any SNP differences and eight plasmids had only one SNP compared to the reference genomes. Only in $S$. Newport that carried the largest plasmid, we detected in one replicate five SNPs. The SNPs between three replicates from one plasmid were never detected on the same base position within the plasmid. In particular, we identified eight instances of addition or deletion occurred in homopolymer regions. There were three instances of a single base insertion/deletion, and a single instance of two basepair deletion. No substitutions were observed. Interestingly, variants appeared disproportionately in AT-rich regions. There were six variants in poly-A or poly- $\mathrm{T}$ regions, but only two variants observed in the poly-G region. Most of the variants, we observed consisted of a deletion or an insertion in a homopolymer; these kinds of errors are known to occur as an artifact of sequencing rather than representing an artifact of the MDA process. 
TABLE 4 | Number of variants observed in genome assemblies between the three replicates.

\begin{tabular}{|c|c|c|c|}
\hline Isolate name & $\begin{array}{l}\text { \# of variance } \\
\text { replicate } 1\end{array}$ & $\begin{array}{l}\text { \# of variance replicate } \\
2\end{array}$ & $\begin{array}{l}\text { \# of variance } \\
\text { replicate } 3\end{array}$ \\
\hline Enteritidis & - & - & - \\
\hline Tennessee & - & - & - \\
\hline Weltefreden & - & - & $\begin{array}{l}\text { add } 1 \mathrm{~A} \text { in } \\
\text { homopolymer } \\
\text { stretch }\end{array}$ \\
\hline Heidelberg & - & - & - \\
\hline \multicolumn{4}{|l|}{ CFSAN002064 } \\
\hline Heidelberg & - & - & add $1 \mathrm{~T}$ in \\
\hline \multirow[t]{2}{*}{ CFSAN002069 } & & & $\begin{array}{l}\text { homopolymer } \\
\text { stretch }\end{array}$ \\
\hline & - & - & - \\
\hline Typhimurium & $\begin{array}{l}\text { del } 1 \mathrm{~A} \text { in } \\
\text { homopolymer } \\
\text { stretch }\end{array}$ & $\operatorname{add} A$ & $\begin{array}{l}\text { add } 1 \mathrm{~A} \text { to } \\
\text { homopolymer } \\
\text { stretch }\end{array}$ \\
\hline Newport & del T & $\begin{array}{l}\text { del AC,add T,del 1G in } \\
\text { homopolymer stretch, } \\
\text { del } 1 \mathrm{~A} \text { in homopolymer } \\
\text { stretch }\end{array}$ & - \\
\hline Agona & - & $\begin{array}{l}\text { del } 1 \mathrm{G} \text { in homopolymer } \\
\text { stretch }\end{array}$ & $\begin{array}{l}\text { add } 1 \mathrm{~A} \text { to } \\
\text { homopolymer } \\
\text { stretch }\end{array}$ \\
\hline \multirow[t]{2}{*}{ Muenster } & - & - & - \\
\hline & - & - & - \\
\hline
\end{tabular}

"Add" indicates addition of a nucleotide, "Del" indicates deletion; no substitution was observed.

\section{CONCLUSION}

Our experiments demonstrate that MDA provides a more efficient way to prepare low copy number plasmids for sequencing: it requires much less time and effort than using electroporation to insert the plasmid inside E. coli in order to increase plasmid numbers (Hoffmann et al., 2017), and our process minimizes amplification bias allowing closure of high molecular weight plasmids. By using a single MDA reaction after plasmid isolation, we were able to produce adequate quantities of high quality plasmid DNA for long read sequencing. We successfully closed 11 plasmids ranging between 37 and $166 \mathrm{~kb}$, isolated from nine different Salmonella serovars. Using MDA, the amount of plasmid DNA produced can be increased 20-fold and enables researchers to conveniently replicate large quantities of low copy number, high molecular

\section{REFERENCES}

Allard, M. W., Strain, E., Melka, D., Bunning, K., Musser, S. M., Brown, E. W., et al. (2016). Practical value of food pathogen traceability through building a whole-genome sequencing network and database. J. Clin. Microbiol. 54, 1975-1983. doi: 10.1128/JCM.00081-16

Bennett, P. M. (2009). Plasmid encoded antibiotic resistance: acquisition and transfer of antibiotic resistance genes in bacteria: plasmid-encoded antibiotic resistance. Br. J. Pharmacol. 153, S347-S357. doi: 10.1038/sj.bjp.0707607

Bleier, S., Maier, P., Allgayer, H., Wenz, F., Zeller, W. J., Fruehauf, S., et al. (2008). Multiple displacement amplification enables large-scale clonal analysis following retroviral gene therapy. J. Virol. 82, 2448-2455. doi: 10.1128/JVI.00584-07 weight plasmid DNA, producing material suitable for sequencing on the PacBio Sequel. Utilizing the multiplexing protocol further maximized the throughput and reduced the cost for plasmid closure. Our method may also be applied to manipulating any other circular DNA, such as mitochondrial DNA or chloroplast DNA.

\section{DATA AVAILABILITY STATEMENT}

The original contributions presented in the study are included in the article/Supplementary Material, further inquiries can be directed to the corresponding author.

\section{AUTHOR CONTRIBUTIONS}

All authors played an integral part of project conception. Each author has read and approved the final version of the manuscript. Specifically, NG-E and MH conceived and designed the experiments. KY performed the experiments. $\mathrm{KY}$ and $\mathrm{MH}$ analyzed the data and wrote the manuscript.

\section{FUNDING}

This project was supported by internal FDA/CFSAN research funding.

\section{ACKNOWLEDGMENTS}

Scientific writing assistance was provided by Lili Fox Vélez, Ph.D., Office of Regulatory Science.

\section{SUPPLEMENTARY MATERIAL}

The Supplementary Material for this article can be found online at: https://www.frontiersin.org/articles/10.3389/fmicb.2021.617487/ full\#supplementary-material

Supplementary Figure 1 | Fragment size on amplified plasmid DNA. The fragment sizes of plasmid DNA acquired after MDA were measured using Fragment Analyzer. The average fragment sizes (in bp) are shown on the top. The amplified products ranged between 35 and $45 \mathrm{~kb}$. 
at: https://www.cdc.gov/narms/pdf/2015-NARMS-Annual-Report-cleared_2508. pdf (Accessed January 22, 2021).

Cheung, V. G., and Nelson, S. F. (1996). Whole genome amplification using a degenerate oligonucleotide primer allows hundreds of genotypes to be performed on less than one nanogram of genomic DNA. Proc. Natl. Acad. Sci. U. S. A. 93, 14676-14679. doi: 10.1073/pnas.93.25.14676

Chin, C.-S., Alexander, D. H., Marks, P., Klammer, A. A., Drake, J., Heiner, C., et al. (2013). Nonhybrid, finished microbial genome assemblies from long-read SMRT sequencing data. Nat. Methods 10, 563-569. doi: 10.1038/nmeth.2474

Darling, A. C., Mau, B., Blattner, F. R., and Perna, N. T. (2004). Mauve: multiple alignment of conserved genomic sequence with rearrangements. Genome Res. 14, 1394-1403. doi: 10.1101/gr.2289704

Dean, F. B., Hosono, S., Fang, L., Wu, X., Faruqi, A. F., Bray-Ward, P., et al. (2002). Comprehensive human genome amplification using multiple displacement amplification. Proc. Natl. Acad. Sci. U. S. A. 99, 5261-5266. doi: 10.1073/pnas.082089499

del Solar, G., Giraldo, R., Ruiz-Echevarria, M. J., Espinosa, M., and Diaz-Orejas, R. (1998). Replication and control of circular bacterial plasmids. Microbiol. Mol. Biol. Rev. 62, 434-464. doi: 10.1128/MMBR.62.2.434-464.1998

Folster, J. P., Pecic, G., Singh, A., Duval, B., Rickert, R., Ayers, S., et al. (2012). Characterization of extended-spectrum cephalosporin-resistant Salmonella enterica serovar Heidelberg isolated from food animals, retail meat, and humans in the United States 2009. Foodborne Pathog. Dis. 9, 638-645. doi: 10.1089/fpd.2012.1130

Hoffmann, M., Pettengill, J. B., Gonzalez-Escalona, N., Miller, J., Ayers, S. L., Zhao, S., et al. (2017). Comparative sequence analysis of multidrug-resistant IncA/C plasmids from Salmonella enterica. Front. Microbiol. 8:1459. doi: 10.3389/fmicb.2017.01459

Hoffmann, M., Zhao, S., Pettengill, J., Luo, Y., Monday, S. R., Abbott, J., et al. (2014). Comparative genomic analysis and virulence differences in closely related Salmonella enterica serotype Heidelberg isolates from humans, retail meats and animals. Genome Biol. Evol. 6, 1046-1068. doi: 10.1093/gbe/evu079

Karp, B. E., Tate, H., Plumblee, J. R., Dessai, U., Whichard, J. M., Thacker, E. L., et al. (2017). National antimicrobial resistance monitoring system: two decades of advancing public health through integrated surveillance of antimicrobial resistance. Foodborne Pathog. Dis. 14, 545-557. doi: 10.1089/ fpd.2017.2283

Krumsiek, J., Arnold, R., and Rattei, T. (2007). Gepard: a rapid and sensitive tool for creating dotplots on genome scale. Bioinformatics 23, 1026-1028. doi: 10.1093/bioinformatics/btm039

Liao, J., Orsi, R. H., Carroll, L. M., Kovac, J., Ou, H., Zhang, H., et al. (2019). Serotype-specific evolutionary patterns of antimicrobial-resistant Salmonella enterica. BMC Evol. Biol. 19:132. doi: 10.1186/s12862-019-1457-5

Lindsey, R. L., Fedorka-Cray, P. J., Frye, J. G., and Meinersmann, R. J. (2009). Inc A/C plasmids are prevalent in multidrug-resistant Salmonella enterica isolates. Appl. Environ. Microbiol. 75, 1908-1915. doi: 10.1128/AEM.02228-08

Lorenz, S. C., Monday, S. R., Hoffmann, M., Fischer, M., and Kase, J. A. (2016). Plasmids from Shiga toxin-producing Escherichia coli strains with rare enterohemolysin gene (ehxA) subtypes reveal pathogenicity potential and display a novel evolutionary path. Appl. Environ. Microbiol. 82, 6367-6377. doi: 10.1128/AEM.01839-16

Pacific-Bioscience (2019). PACBIO SEQUEL SYSTEMS-PRODUCTS + SERVICES. Available at: https:/www.pacb.com/products-and-services/sequel-system/ (Accessed January 22, 2021).

Scallan, E., Hoekstra, R. M., Angulo, F. J., Tauxe, R. V., Widdowson, M. A., Roy, S. L., et al. (2011). Foodborne illness acquired in the United Statesmaj pathogens. Emerg. Infect. Dis. 17, 7-15. doi: 10.3201/eid1701.P11101

Spits, C., Le Caignec, C., De Rycke, M., Van Haute, L., Van Steirteghem, A., Liebaers, I., et al. (2006). Whole-genome multiple displacement amplification from single cells. Nat. Protoc. 1, 1965-1970. doi: 10.1038/nprot.2006.326

Conflict of Interest: The authors declare that the research was conducted in the absence of any commercial or financial relationships that could be construed as a potential conflict of interest.

Copyright () 2021 Yao, González-Escalona and Hoffmann. This is an open-access article distributed under the terms of the Creative Commons Attribution License (CC BY). The use, distribution or reproduction in other forums is permitted, provided the original author(s) and the copyright owner(s) are credited and that the original publication in this journal is cited, in accordance with accepted academic practice. No use, distribution or reproduction is permitted which does not comply with these terms. 